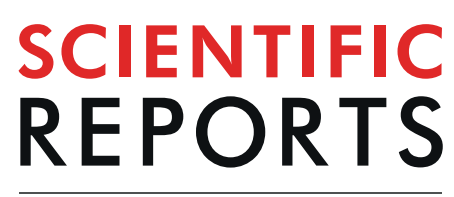

\title{
Decreased expression of femXAB genes and fnbp mediated biofilm pathways in OS-MRSA clinical isolates
}

\author{
Umarani Brahma ${ }^{1}$, Paresh Sharma ${ }^{1}$, Shweta Murthy ${ }^{1}$, Savitri Sharma ${ }^{2}$, Shalini Chakraborty ${ }^{1}$, \\ Sundarapu Naga Appalaraju ${ }^{1} \&$ Vasundhra Bhandari $^{1^{*}}$
}

Methicillin-Resistant Staphylococcus aureus (MRSA) is a significant threat to human health. Additionally, biofilm forming bacteria becomes more tolerant to antibiotics and act as bacterial reservoir leading to chronic infection. In this study, we characterised the antibiotic susceptibility, biofilm production and sequence types (ST) of 74 randomly selected clinical isolates of $S$. aureus causing ocular infections. Antibiotic susceptibility revealed $74 \%$ of the isolates as resistant against one or two antibiotics, followed by $16 \%$ multidrug-resistant isolates (MDR), and $10 \%$ sensitive. The isolates were characterized as MRSA $(n=15)$, Methicillin-sensitive $S$. aureus (MSSA, $n=48$ ) and oxacillin susceptible mecA positive $S$. aureus (OS-MRSA, $n=11$ ) based on oxacillin susceptibility, mecA gene PCR and PBP2a agglutination test. All OS-MRSA would have been misclassified as MSSA on the basis of susceptibility test. Therefore, both phenotypic and genotypic tests should be included to prevent strain misrepresentation. In addition, in-depth studies for understanding the emerging OS-MRSA phenotype is required. The role of fem $X A B$ gene family has been earlier reported in OS-MRSA phenotype. Sequence analysis of the fem $X A B$ genes revealed mutations in fem $\times(\mathrm{K} 3 \mathrm{R}, \mathrm{H} 11 \mathrm{~N}, \mathrm{~N} 18 \mathrm{H}$ and I51V) and fem $B$ (L410F) genes. The fem $X A B$ genes were also found down-regulated in OS-MRSA isolates in comparison to MRSA. In OS-MRSA isolates, biofilm formation is regulated by fibronectin binding proteins A \& B. Molecular typing of the isolates revealed genetic diversity. All the isolates produced biofilm, however, MRSA isolates with strong biofilm phenotype represent a worrisome situation and may even result in treatment failure.

Staphylococcus aureus causes a wide range of diseases in both humans and animals ${ }^{1}$. It is an opportunistic pathogen, known to acquire resistance against several classes of antibiotics. Methicillin-Resistant S. aureus (MRSA) is defined as strains with an oxacillin minimum inhibitory concentration (MIC) of $\geq 4 \mathrm{mg} / \mathrm{L}^{2}$. Most of the MRSA strains contain the mecA gene located on the staphylococcal cassette chromosome mec (SCCmec) which encodes for a modified penicillin-binding protein $2 \mathrm{a}(\mathrm{PBP} 2 \mathrm{a})^{2}$. MRSA has emerged as a huge threat around the globe as community, hospital, and livestock acquired infections ${ }^{2,3}$. The surveillance network in the United States claims the MRSA increase from $29.5 \%$ in 2000 to $41.6 \%$ in $2005^{4}$. Whereas a report in 2013 from India showed an increase in MRSA prevalence among non-ICU inpatients and ICU patients to be 42 and 43\%, respectively in 2008 as compared to 49 and $47 \%$, in 2009, respectively ${ }^{5}$. There is also an increasing prevalence of MRSA in ocular infections which is alarming ${ }^{4,6,7}$. Reports suggest MRSA infections affect the neonates and aged individuals at a more aggressive rate because of their comparatively decreased immunity ${ }^{8,9}$ common surgeries such as photo reactive or refractive keratectomies often are paving the way for MRSA infectious keratitis making it an imperceptibly tricky situation for the patients and doctors alike ${ }^{10,11}$. Another recent unnerving revelation is that $S$. aureus may disseminate from the blood to eye in patients suffering from bacteraemia ${ }^{12}$.

Recently, OS-MRSA (Oxacillin susceptible mecA positive S. aureus) clinical isolates have surfaced across the globe which possess the mecA gene but are susceptible to oxacillin ${ }^{2,3}$. However, not much data is yet available on 
the prevalence, virulence, resistance mechanism of OS-MRSA strains and their impact on the current treatment regimen. Another imperative should be the molecular characterisation of existing clinical isolates.

In addition to the intriguing phenotype of $S$. aureus, their capacity to form biofilms on biotic or abiotic surfaces especially, medical devices, catheter, ocular implants and corneal stroma further magnify the problem ${ }^{13}$. Biofilm formation is an innate component of the prokaryotic life cycle which ensures the survival of the bacterial cells by fending off host immune attacks as well as external therapeutic/antibiotics and also promote dispersal to other uninfected parts thereby maintaining a chronic infection ${ }^{14}$. We hence need to identify and understand the molecular characteristics of such bacterial populations to unarm them. In the current study, we are trying to understand the molecular characteristic, resistance and biofilm profiles of MRSA and MSSA strains isolated from ocular infections and to determine the prevalence of OS-MRSA which is not well understood.

\section{Materials and Methods}

Isolation of S. aureus from clinical samples. Clinical isolates of randomly selected S. aureus were received from L.V Prasad Eye Institute (LVPEI), Hyderabad, India. Further, species identification and characterisation of $S$. aureus cultures were performed using various biochemical and molecular tests as discussed earlier ${ }^{3}$. Following an overnight incubation in trypticase soy broth (TSB) at $37^{\circ} \mathrm{C}$, the cultures were streaked on Baird Parker agar plates (30\% egg yolk emulsion and $1 \%$ potassium tellurite) and after that onto mannitol salt agar plates. Yellow colonies on the plates were considered as $S$. aureus and inoculated further in TSB for other biochemical tests such as catalase, Latex agglutination, Coagulase test and Gram staining. Species confirmation was also done by sequence analysis of the amplified PCR product of $16 S \mathrm{rRNA}$ gene as mentioned below.

PCR amplification. Genomic DNA was isolated using the Wizard genomic kit (Promega) as per manufacturer's instructions. Briefly, the overnight grown culture in TSB media was centrifuged at $3200 \times \mathrm{g}$ for 10 minutes and washed twice in PBS. Cells were pelleted and resuspended in $500 \mu \mathrm{l}$ lysis buffer $(50 \mathrm{mM}$ EDTA) and $50 \mu \mathrm{l}$ of lysozyme $(20 \mathrm{mg} / \mathrm{ml})$ and incubated at $37^{\circ} \mathrm{C}$ for $2 \mathrm{hrs}$. PCR was performed for $16 S \mathrm{rRNA}$, mecA, mecC and SCCmec types and agr typing as described previously ${ }^{3,15-18}$.

Multilocus sequence typing (MLST). Internal fragments of seven housekeeping genes, Carbamate kinase $(a r c)$, Shikimate dehydrogenase ( $a r o E)$, Glycerol kinase $(g l p F)$, Guanylate kinase $(g m k)$, Phosphate acetyltransferase ( $p t a)$, Triosephosphate isomerase $(t p i)$, and Acetyl coenzyme A acetyltransferase ( $y q i L)$ were amplified using the approach described on http://www.mlst.net/. The sequence types (STs) were designated by comparing the data of seven sequenced internal fragments of each strain with the S. aureus MLST database (http://www. mlst.net/).

Global optimal eBURST (goeBURST) of identified STs, was analysed using the PHYLOViZ online software ${ }^{19}$. S. aureus MLST public database was used to make the minimum spanning tree based on the goeBURST analysis for the identified STs. The nLocus Variant (nLV) was kept to 2 to keep all STs with a distance equal to or below 2 connected in the tree.

Antibiotic susceptibility profile. The antibiotic susceptibility profile was determined using the disk diffusion method and microbroth dilution assay as per CLSI guidelines with minor modifications as mentioned before $^{18}$. Briefly, the overnight grown culture was adjusted to $0.5 \mathrm{McF}$ arland standard (Himedia, Mumbai, India) and was streaked onto Mueller-Hinton Agar (Himedia, Mumbai, India) plates for disk diffusion while Mueller-Hinton broth was used for micro-broth dilution assay. The disk diffusion assay was done against tetracycline $(30 \mu \mathrm{g})$, gentamicin $(10 \mu \mathrm{g})$, rifampicin $(5 \mu \mathrm{g})$, erythromycin $(15 \mu \mathrm{g})$, ciprofloxacin $(10 \mu \mathrm{g})$, clindamycin $(2 \mu \mathrm{g})$, and cefoxitin $(30 \mu \mathrm{g})$. Resazurin dye based microbroth dilution assay was performed against oxacillin, vancomycin and linezolid ${ }^{18}$. ATCC 29213 (methicillin sensitive control strain) and ATCC 700699 (methicillin-resistant control strain with reduced vancomycin susceptibility) were used as a control for these assays $^{3,18}$. Clinical isolates showing resistance against three or more class of antibiotics were described as multidrug-resistant (MDR), resistance against one or two class of antibiotic was referred as resistant and sensitivity towards all antibiotics was mentioned as pan-sensitive in the result section.

PBP2a Latex Agglutination Test. PBP2a, which is the mecA gene product was detected using Oxoid PBP2' Latex Agglutination Test' kit (DR0900A, Thermo Scientific ${ }^{\mathrm{TM}}$ ), following the manufacturer's instructions. ATCC 43300 and ATCC 29213 were used as controls.

Sequence analysis of fem $X A B$ family. The $f e m X A B$ gene family was amplified as described earlier ${ }^{20}$. The primers used for amplification of $f e m$ genes were as follows: $f e m X$ (For-GCCATGGAAAAGATGCATATCAC and Rev-CTCGAGTTTTCGTTTTAATTTACGAG), femA (For-GGATCCATGAAGTTTACAAATTTAACAGC Rev-CTCGAGAAAAATTCTGTCTTTAAGTTTTTTAAG) and femB (GGATCCATGAAGTTT ACAAATTTAACAGC and Rev-CTCGAGTTTCTTTAATTTTTTACGTAATTTATCG $)^{20}$. The amplified PCR product was sequenced using forward and reverse primer along with the internal primers for all the respective genes (Internal femA-GATCCGTGCTACAAATTCGT; internal femX-CACCAATTGATAAAAATGATG and internal femB-CAACTGAG TATGATACATCGA). The data was analysed for mutation using DNA star software (SeqMan NGen ${ }^{\circledR}$. Version 12.0. DNASTAR. Madison, WI.).

Expression analysis of fem $X A B$ and biofilm-related genes. RNA was isolated using the Macherey Nagel RNA isolation kit as per the manufacturer's instructions (NucleoSpin ${ }^{\circledR}$ RNA, 740955.10). Approximately from $2.5 \mu \mathrm{g}$ of RNA, cDNA was synthesised using the Clontech cDNA synthesis kit (PrimeScript ${ }^{\mathrm{TM}} 1$ st strand cDNA Synthesis Kit, 6110B). SYBR green-based relative gene expression analysis was carried out using the comparative $2^{-\Delta \Delta} C_{t}$ method using the ABI real-time detection system (7500, ABI). Real-time PCR was performed for 


\begin{tabular}{|c|c|c|c|}
\hline S. No & Primer & Forward sequence $\left(5^{\prime}-3^{\prime}\right)$ & Reverse sequence $\left(5^{\prime}-3^{\prime}\right)$ \\
\hline 1. & gyrB & CCAGGTAAATTAGCCGATTGC & AAATCGCCTGCGTTCTAGAG \\
\hline 2. & icaA & GAGGTAAAGCCAACGCACTC & CCTGTAACCGCACCAAGTTT \\
\hline 3. & $i c a B$ & ATACCGGCGACTGGGTTTAT & TTGCAAATCGTGGGTATGTGT \\
\hline 4. & $i c a C$ & CTTGGGTATTTGCACGCATT & GCAATATCATGCCGACACCT \\
\hline 5. & $i c a D$ & ACCCAACGCTAAAATCATCG & GCGAAAATGCCCATAGTTTC \\
\hline 6. & fnbp A & AAATTGGGAGCAGCATCAGT & GCAGCTGAATTCCCATTTTC \\
\hline 7. & fnbp B & GCAGCTGAATTCCCATTTTC & ACCTTCTGCATGACCTTCTGCACCT \\
\hline 8. & femA & TCGATCCATATTTACCATATCAATACTTG & TCCTAAGTTACTCATTTTATCAAAGAAC \\
\hline 9. & femB & TCGTGCCATTTGAAGGTCG & TCAAGGTTTAATACGCCCATCC \\
\hline 10. & femX & GCGAAGAATCGCTGTAGGTC & TGCATACGCTTTCTCAGCTT \\
\hline
\end{tabular}

Table 1. List of real-time PCR primers used in the study.

\begin{tabular}{|l|l|l|}
\hline Antibiotic & $\begin{array}{l}\text { Number of resistant } \\
\text { isolates (\%) }\end{array}$ & $\begin{array}{l}\text { Number of sensitive } \\
\text { isolates (\%) }\end{array}$ \\
\hline Oxacillin/Cefoxitin & $15(20)$ & $59(80)$ \\
\hline Linezolid & $0(0)$ & $74(100)$ \\
\hline Gentamycin & $17(23)$ & $57(77)$ \\
\hline Erythromycin & $37(50)$ & $37(50)$ \\
\hline Rifampicin & $4(5)$ & $70(95)$ \\
\hline Tetracycline & $9(12)$ & $65(88)$ \\
\hline Clindamycin & $7(9)$ & $67(91)$ \\
\hline Ciprofloxacin & $63(85)$ & $11(15)$ \\
\hline Vancomycin & $0(0)$ & $74(100)$ \\
\hline
\end{tabular}

Table 2. Overall susceptibility profile of the clinical isolates against tested antibiotics.

fem $X A B$ genes and biofilm-related genes (icaADBC and $f n b p A \& B$ ). The gyrB gene was used as internal control in the study. All the primers used in the study are mentioned in Table 1. All the experiments were repeated thrice.

Biofilm profiling by crystal violet assay. The biofilm forming capacity of each strain was determined using crystal violet (CV) assay as described previously, ATCC 29213 and ATCC 33592 were used as controls ${ }^{21}$. Briefly, the overnight culture was diluted in TSB (1:100), and dispensed in 96 well plate (200 $\mu \mathrm{l} /$ well). The wells with only broth were treated as the control. After incubation for $18-24 \mathrm{hrs}$ at $37^{\circ} \mathrm{C}$, the plate was washed 3 times with PBS to remove planktonic cells or media particles. Methanol was added to each well for 15 minutes and then air-dried for 30 minutes. $0.1 \%$ crystal violet solution was added to each well and incubated at room temperature for 20 minutes followed by washing using distilled water. After washing, 33\% acetic acid was added to each well and absorbance was taken at $590 \mathrm{~nm}$. Mean absorbance values of each sample was calculated and compared with the mean values of controls. All experiments were repeated thrice in quadruplicates. The data were analyzed as described before ${ }^{22}$.

The following conditions were used to interpret the results:

$$
\begin{aligned}
& \text { O. Dc } \geq \text { O.Ds }=>\text { non }- \text { adherent. } \\
& \text { O.Dc }<\text { O.Ds } \leq 2 * \text { O.Dc }=>\text { weakly adherent. } \\
& 2 * \text { O.Dc }<\text { O.Ds } \leq 4 * \text { O.Dc }=>\text { moderately adherent. } \\
& 4 * \text { O.Dc }<\text { O.Ds }=>\text { strongly adherent. } \\
& \text { [O.Dc }=>\text { Optical Density of control; O.Ds }=>\text { Optical Density of sample] }
\end{aligned}
$$

Congo-Red assay. Congo red plates were prepared using Brain Heart infusion agar ( $52 \mathrm{gm} / \mathrm{L})$, sucrose $(50 \mathrm{gm} / \mathrm{L})$, congo-red stain $(0.8 \mathrm{gm} / \mathrm{ml})$. Overnight cultures of the samples in TSB was inoculated in MHB and kept in the incubator-shaker till they reached an O.D $D_{600 \mathrm{~nm}}$ of $0.08-0.13$. The cultures were streaked on congo-red agar plates and incubated for 16-18 hours. Plates with black colonies were considered as Congo red positive.

\section{Results}

Antibiotic susceptibility profile. The antibiotic susceptibility assays were performed for 74 clinical isolates of S. aureus against 10 antibiotics (Table 2). High resistance rates were observed against ciprofloxacin (85\%) and erythromycin (50\%). Moderate resistance was observed against gentamicin (23\%) and oxacillin/cefoxitin (20\%). However, low resistance was observed against tetracycline (12\%), clindamycin (9\%) and rifampicin (5\%). All the clinical isolates were susceptible to vancomycin and linezolid. Overall, $16 \%$ of the isolates were multidrug resistant followed by $74 \%$ resistant, and $10 \%$ pan-sensitive isolates. 


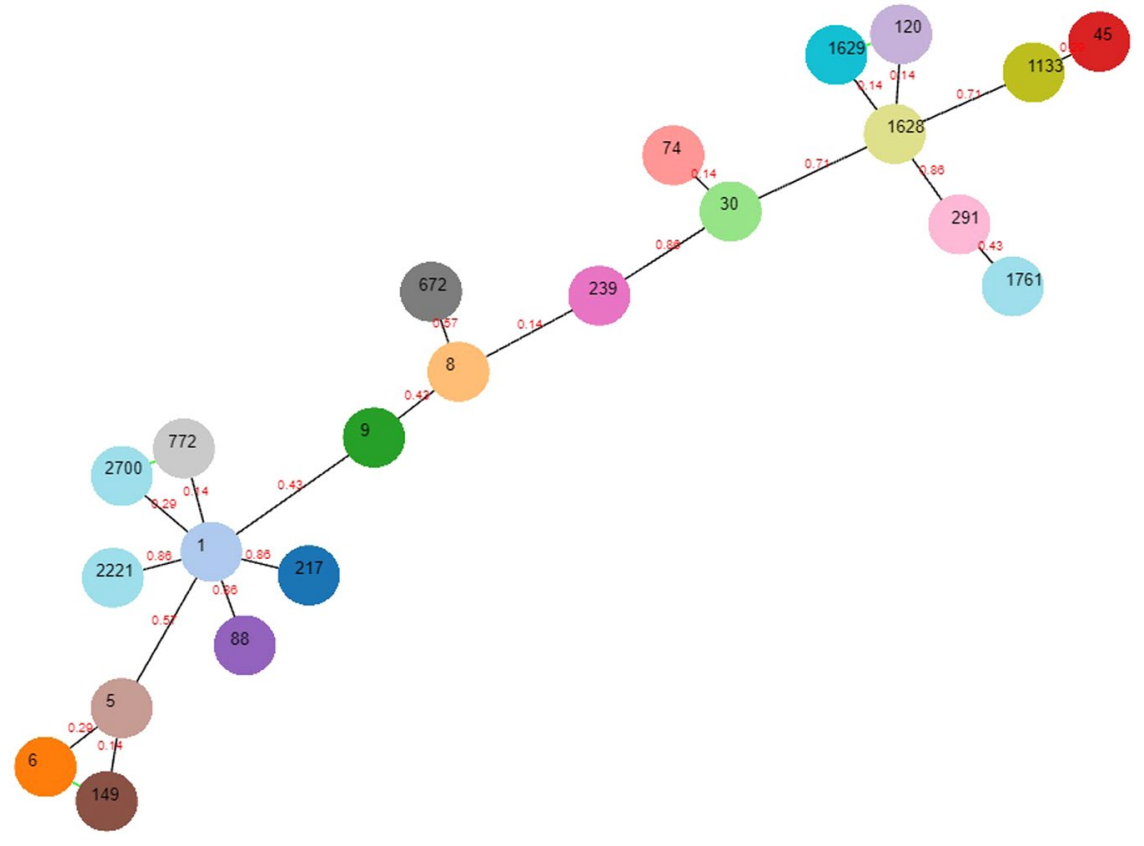

Figure 1. Phylogenetic relationship among 74 clinical isolates of Staphylococcus aureus using Sequence types (ST) obtained by Multi locus Sequence typing (MLST).

Different STs of S. aureus persists in ocular infections. MLST analysis of the 74S. aureus clinical isolates determined 22 different sequence types (STs). ST1 (13.51\%) was most common followed by ST672 (12.16\%), ST217 (10.81\%), ST120 (8.10\%), ST30 (6.75\%), ST239 (6.75\%), ST5 (5.40\%), ST8 (5.40\%), ST1628 (5.40\%), ST291 (4.05\%), ST772 (4.05\%), ST6 (2.70\%) and ST9 (2.70\%). Two major groups were identified using the goeBURST analysis (Fig. 1). The first and major group was around ST1 which was surrounded by 8 different STs while the second cluster was around ST1628. Rest of the STs were in singletons or doubletons indicating significant differences between these strains.

Agr type I and III were predominant. Agr typing of the 74 clinical isolates showed type I $(\mathrm{n}=32)$ and III $(\mathrm{n}=18)$ to be the major types, followed by types IV $(\mathrm{n}=12)$ and II $(\mathrm{n}=10)$. One isolate displayed mixed type $(\mathrm{I}+\mathrm{II})$, and one was non-typeable.

Clinical isolates of $S$. aureus exhibited strong, moderate and weak biofilm forming capacity. Biofilm forming capacity of the 74 clinical isolates was determined using CV assay. The majority (55\%) of the isolates exhibited moderate biofilm forming capacity followed by $39 \%$ isolates which manifested strong biofilm production. Only $6 \%$ of the isolates formed weak biofilms. All the isolates formed black colonies on congo red agar plates.

Characterisation of clinical isolates of MRSA. Antibiotic susceptibility assays against oxacillin and cefoxitin determined 15 isolates as methicillin-resistant (Table 2). Twelve MRSA clinical isolates were multi-drug resistant, and the remaining 3 were resistant to one or two classes of antibiotics. In 2 isolates, the mec $A$ gene was not amplified, but they exhibited oxacillin resistance (MIC $>4 \mathrm{mg} / \mathrm{L}$ ). Therefore, the SCCmec typing of the 13 isolates revealed 7 as type III, followed by 3 isolates as type V, 2 isolates as IVc and 1 isolate was non-typeable (Table S1). Molecular typing of MRSA isolates revealed ST239 (33\%) as the major sequence type followed by ST217 (26.66\%). ST772 (13.33\%), ST1 (6.66\%), ST8 (6.66\%), ST9 (6.66\%), ST5 (6.66\%) represent smaller proportions. Agr typing of these strains showed $66.67 \%$ isolates belonged to type I followed by $26.67 \%$ of type II and $6.67 \%$ of type III.

Majority of the MRSA isolates (60\%) formed strong biofilm, followed by $33 \%$ moderate and only $6.6 \%$ of the isolates formed weak biofilm as determined by CV assay.

Prevalence of Oxacillin susceptible mecA positive (OS-MRSA) isolates and role of fem XAB gene family. Eleven $S$. aureus clinical isolates which were susceptible to oxacillin and cefoxitin and also positive for $m e c A$ gene and PBP2a agglutination were characterized as OS-MRSA (Table S1). Eighty one percent of the isolates were found resistant to one or two classes of antibiotic, and 18.2\% were pan-sensitive. Sequence analysis of fem XAB genes in OS-MRSA isolates showed mutations in fem $\times(\mathrm{K} 3 \mathrm{R}, \mathrm{H} 11 \mathrm{~N}, \mathrm{~N} 18 \mathrm{H}$ and I51V) and fem $B$ (L410F) gene, however, no mutations were found in fem $A$. Gene expression analysis revealed decreased expression of all the fem genes in comparison to MRSA (Fig. 2).

OS-MRSA isolates were genetically diverse and they belonged to 10 different STs represented by ST 30, ST120, ST772, ST1133, ST1, ST5, ST14, ST45, ST88, and ST217. SCCmec typing revealed Type V $(\mathrm{n}=3)$, IVc $(\mathrm{n}=2)$, IVa 


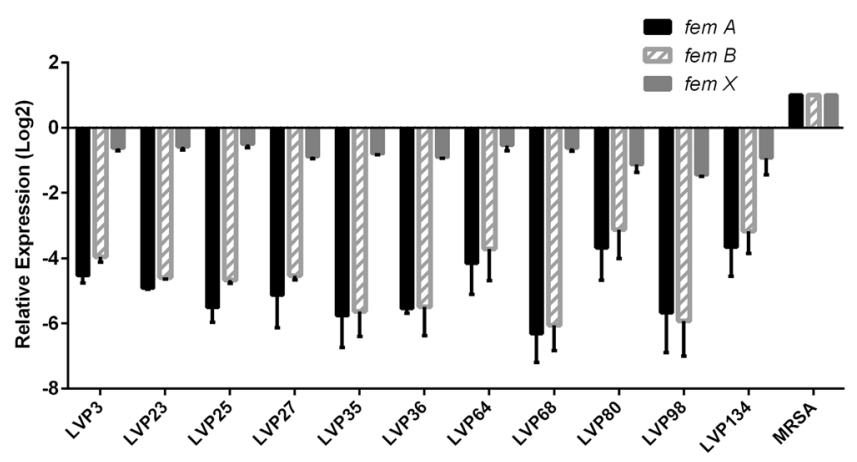

Figure 2. Relative gene expression analysis of the $f e m X A B$ family of genes in 11 OS-MRSA isolates using realtime PCR. The bar graph represents relative gene expression $\left(\log _{2} \pm \mathrm{SD}\right)$.

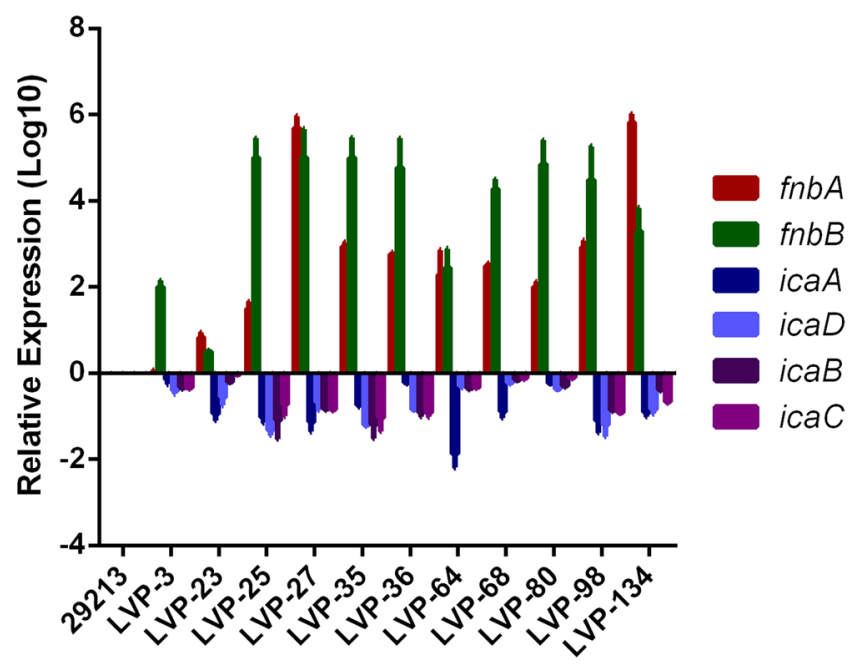

Figure 3. Relative gene expression analysis of biofilm pathway related genes, icaADBC and fibronectin binding proteins A \& B in 11 OS-MRSA isolates. The bar graph represents gene expression levels $\left(\log _{10} \pm S D\right)$.

$(\mathrm{n}=1)$, III $(\mathrm{n}=2)$ and II $(\mathrm{n}=2)$ in the group and one non-typeable. Major agr types appear to be III (54.5\%) and I (18.2\%), followed by type IV (18.2\%) while one isolate was non-typeable (5\%). CV assay revealed $45.45 \%$ to be moderate, and strong biofilm producers followed by $9.09 \%$ to be weak biofilm producer (Table S1). Gene expression analysis of $f n b$ and ica genes involved in biofilm formation revealed up-regulation of $f n b A$ and $B$ genes in all OS-MRSA isolates whereas icaABCD genes were down-regulated (Fig. 3).

Characterization of Methicillin Sensitive S. aureus (MSSA). Isolates with oxacillin MIC of $\leq 2 \mathrm{mg} / \mathrm{L}$ were categorized as methicillin-sensitive $S$. aureus (MSSA, $\mathrm{n}=48$ ) (Table 2). MSSA isolates were genetically diverse with 17 different STs type represented by ST672, ST1, ST120, ST1628, ST8, ST291, ST217, ST5, ST6, ST30, ST1629, ST1761, ST2700, ST2221, ST74, ST9 and ST149.

Majority of the isolates belonged to agr type I (39.6\%) and III (25\%) followed by types IV (20.8\%), II (10.4\%), and type I \& II (2.08\%) (Table S1). Predominantly MSSA isolates showed moderate biofilm (64.6\%) formation followed by strong $(31.25 \%)$ and weak $(4.17 \%)$ biofilm phenotype.

\section{Discussion}

The present study represents the molecular variability and antibiotic susceptibility profiles of $S$. aureus clinical isolates causing ocular infections. Antibiotic susceptibility determined majority of the isolates as resistant to one or two classes of antibiotics, followed by MDR and pan sensitive clinical isolates. These clinical isolates were further characterized as methicillin-sensitive or resistant based on the phenotypic and genotypic tests. MRSA in ocular infections had been previously reported from countries such as Taiwan (57.6\%), China (52.8\%), India (43\%), United States $(41.6 \%)$ and Brazil $(9.9 \%)^{2,23-26}$. We found $17.6 \%$ MRSA $(\mathrm{n}=15)$ in our study and two MRSA without the $m e c A$ gene, which is in line with previous findings where $9.8 \%$ of MRSA isolates were $m e c A$ negative ${ }^{27}$.

The present finding also highlighted a $14.86 \%$ prevalence rate of OS-MRSA clinical isolates which were not reported earlier from ocular infections in India. OS-MRSA has surfaced across the globe and reported from human and animal infections $s^{2,3}$. We also found 9 oxacillin susceptible clinical isolates which did not produce PBP2a but were mecA PCR positive ${ }^{28}$. OS-MRSA can be misinterpreted if only either phenotypic or genotypic test is used and may have serious implications ${ }^{29}$. Earlier reports of OS-MRSA have shown mutations in the fem XAB genes ${ }^{20,30}$. 
Consistent with the previous finding by Giannouli et al., 2010, we found mutations in the femX (K3R, H11N, and $\mathrm{N} 18 \mathrm{H}$ ) and the $f e m B(\mathrm{~L} 410 \mathrm{~F})$ gene. An additional new mutation in the $f e m X$ gene (I51V) was identified. The downregulation of $f e m X A B$ genes was seen, in contrast to earlier observation where no change has been reported $^{20}$. Methicillin resistance is caused by changes associated with genes involved in cell wall metabolism and the fem gene family are also involved in cell wall biosynthesis, hence, decreased expression of the fem genes may lead to oxacillin sensitivity in case of OS-MRSA isolates ${ }^{31}$. Increased expression of the femA gene has been reported in high-level MRSA isolates which were comparatively found to be reduced in low-level MRSA followed by MSSA isolates ${ }^{32}$. Therefore, our study indicates the decreased expression of the fem genes, may be responsible for hypersusceptibility towards oxacillin in OS-MRSA isolate ${ }^{20}$. However, the underlying mechanism operative in OS-MRSA is still obscure and need investigation.

Further, molecular typing of the clinical isolates showed diversity among them. We found ST239 is a predominant lineage in MRSA isolates followed by ST217 and ST772 $2^{33-35}$. The ST239 clinical isolates were mostly reported in hospital-associated infections from India and other Asian countries, while ST217 and ST772 were reported from community-acquired MRSA infections ${ }^{36,37}$. ST772 is mainly reported from the southern part of India and is being considered to be replacing ST239 in hospitals ${ }^{37}$. Recently, ST217 has been identified as a new emerging clone in both hospital and community-acquired infections from India ${ }^{37}$. Along with their resistant phenotype, the add-on capacity to form biofilms assist their prolonged survival by enhanced tolerance against host mechanisms or antibiotics ${ }^{14,38}$. Therefore, we also studied the biofilm forming capacity of the clinical isolates with variable methicillin phenotype in an attempt to establish any link with strain lineages and phenotype.

The majority of the MRSA formed strong biofilm $(n=9)$, and predominantly belonged to ST239 $(n=5)$ with SCCmec type III $(\mathrm{n}=4)$ and V $(\mathrm{n}=1)$. ST239 MRSA causing ocular infections have been previously reported to be from Taiwan (29.4\%), although its biofilm phenotype was not evaluated ${ }^{26}$. Strong biofilm forming ST239 isolates were also reported from other countries like Korea, Brazil, and India ${ }^{39-41}$. We also came across ST772 MRSA with SCCmec type $\mathrm{V},(\mathrm{n}=2)$ which formed a strong biofilm and has been considered as an emerging threat responsible for disease outbreaks ${ }^{42}$. One of the mecA negative MRSA belonging to ST5, also formed a strong biofilm. The present findings suggest that MRSA isolates with SCCmec types III and V may be dominant biofilm producers. A recent study shows that SCCmec types III \& V display higher adhesion and are less hydrophilic in comparison to SCCmec type $\mathrm{II}^{40}$. However, there can be a difference in the observed phenomenon when compared in vivo. Biofilm formation have been known to be dependent on icaADBC operon in MSSA isolate, whereas in MRSA, $f n b$ genes are involved ${ }^{43}$. The biofilm mechanism in OS-MRSA isolates has not been studied earlier. Our data reveal that biofilm formation in OS-MRSA isolates is icaADBC independent and regulated via fnb genes.

Agr is studied mostly as virulence locus but recently shown to be involved in biofilm formation ${ }^{44}$. Genotyping and transcriptional analysis to establish a direct correlation between biofilm formation and agr have been done in recent times ${ }^{44}$. In contradiction to the expected decreased biofilm forming capacity of isolates with agr type I, we found isolates (5 MRSA; 2MSSA) with agr type I forming strong biofilms ${ }^{44}$. Therefore, it would be interesting to study such isolates and understand their mechanism of biofilm production. Isolates with type III agr are known to induce moderate biofilms, however, our type III agr isolates showed strong biofilm phenotype ${ }^{44}$. Similar to previous findings, MRSA isolates belonging to ST772/SCCmec type V and agr type II and a mecA negative, ST5 isolates produced strong biofilms ${ }^{44}$. Our findings interestingly support the recent reports where methicillin resistance effects icaABCD and agr phenotype thereby altering expected biofilm phenotype ${ }^{43,45}$. Also 12 out of 15 MRSA isolates were MDR out of which 8 were forming strong biofilms. A recent publication also stated that $87 \%$ of hospital-acquired ocular MRSA infection were $\mathrm{MDR}^{46}$. This is an alarming situation because treating a MDR bacteria forming strong biofilm makes the treatment extremely difficult.

OS-MRSA isolates with strong biofilm $(\mathrm{n}=5)$ belonged to variable ST types, but all belonged to agr type III. There seems to be hardly any study on the biofilm phenotype of the newly emerging class of OS-MRSA isolates, and hence this could shed some light on our understanding of these isolates. OS-MRSA isolates which could be misinterpreted as sensitive isolates with an add-on ability to form strong biofilm may pose a dangerous threat to public health. Another fact to note was that MSSA isolates belonging to agr IV and III appeared to form a strong biofilm.

Our data suggest no association of biofilm intensity with their antibiotic susceptibility phenotype. However, the variable biofilm forming ability might be linked with the differences in their metabolic and biosynthetic processes as evident in the case of Enterococcus faecalis isolates ${ }^{47}$. Hence, it will be interesting to do comparative studies exploring the biofilm mechanism among strong, moderate and weak biofilm isolates which may help us in identifying drug targets against biofilm-forming bacteria. However, any resistant strain with strong biofilm forming capacity will be an immense threat to patients with ocular infections and in other diseases too. Further, with the increasing prevalence of OS-MRSA strains in various infections across the globe, studies are warranted to understand this peculiar phenotype.

Received: 6 October 2018; Accepted: 1 October 2019;

Published online: 05 November 2019

\section{References}

1. Tong, S. Y. C., Davis, J. S., Eichenberger, E., Holland, T. L. \& Fowler, V. G. Staphylococcus aureus Infections: Epidemiology, Pathophysiology, Clinical Manifestations, and Management. Clinical Microbiology Reviews 28, 603-661 (2015).

2. Conceição, T., Coelho, C., de Lencastre, H. \& Aires-de-Sousa, M. Frequent occurrence of oxacillin-susceptible mecA -positive Staphylococcus aureus (OS-MRSA) strains in two African countries: Table 1. Journal of Antimicrobial Chemotherapy dkv261, https:// doi.org/10.1093/jac/dkv261 (2015).

3. Mistry, H. et al. Prevalence and Characterization of Oxacillin Susceptible mecA-Positive Clinical Isolates of Staphylococcus aureus Causing Bovine Mastitis in India. PLOS ONE 11, e0162256 (2016). 
4. Asbell, P. A., Sahm, D. F., Shaw, M., Draghi, D. C. \& Brown, N. P. Increasing prevalence of methicillin resistance in serious ocular infections caused by Staphylococcus aureus in the United States: 2000 to 2005. Journal of Cataract \& Refractive Surgery 34, 814-818 (2008).

5. Joshi, S. Methicillin resistant Staphylococcus aureus (MRSA) in India: Prevalence \& susceptibility pattern. Indian Journal of Medical Research 137, 363-369 (2013).

6. Freidlin, J. et al. Spectrum of Eye Disease Caused by Methicillin-Resistant Staphylococcus Aureus. American Journal of Ophthalmology 144, 313-315 (2007).

7. Adebayo, A. et al. Shifting trends in in vitro antibiotic susceptibilities for common bacterial conjunctival isolates in the last decade at the New York Eye and Ear Infirmary. Graefe's Archive for Clinical and Experimental Ophthalmology 249, 111-119 (2011).

8. Shanmuganathan, V. A., Armstrong, M., Buller, A. \& Tullo, A. B. External ocular infections due to methicillin-resistant Staphylococcus aureus (MRSA). Eye 19, 284-291 (2005).

9. Pak, K. Y., Kim, S. I. \& Lee, J. S. Neonatal Bacterial Conjunctivitis in Korea in the 21st Century:. Cornea 36, 415-418 (2017).

10. Donnenfeld, E. D. et al. Infectious keratitis after photorefractive keratectomy. Ophthalmology 110, 743-747 (2003).

11. Solomon, R. et al. Methicillin-Resistant Staphylococcus aureus Infectious Keratitis Following Refractive Surgery. American Journal of Ophthalmology 143, 629-634 (2007).

12. Jung, J. et al. Incidence and Risk Factors of Ocular Infection Caused by Staphylococcus aureus Bacteremia. Antimicrobial Agents and Chemotherapy 60, 2012-2017 (2016).

13. Bispo, P., Haas, W. \& Gilmore, M. Biofilms in Infections of the Eye. Pathogens 4, 111-136 (2015).

14. Archer, N. K. et al. Staphylococcus aureus biofilms: Properties, regulation, and roles in human disease. Virulence 2, 445-459 (2011).

15. Lina, G. et al. Bacterial Competition for Human Nasal Cavity Colonization: Role of Staphylococcal agr Alleles. Applied and Environmental Microbiology 69, 18-23 (2003).

16. Zhang, K., McClure, J.-A., Elsayed, S., Louie, T. \& Conly, J. M. Novel Multiplex PCR Assay for Characterization and Concomitant Subtyping of Staphylococcal Cassette Chromosome mec Types I to V in Methicillin-Resistant Staphylococcus aureus. Journal of Clinical Microbiology 43, 5026-5033 (2005).

17. Stegger, M. et al. Rapid detection, differentiation and typing of methicillin-resistant Staphylococcus aureus harbouring either mecA or the new mecA homologue mecALGA251. Clinical Microbiology and Infection 18, 395-400 (2012).

18. Mahato, S. et al. Identification of Variable Traits among the Methicillin Resistant and Sensitive Coagulase Negative Staphylococci in Milk Samples from Mastitic Cows in India. Frontiers in Microbiology 8 (2017).

19. Ribeiro-Gonçalves, B., Francisco, A. P., Vaz, C., Ramirez, M. \& Carriço, J. A. PHYLOViZ Online: web-based tool for visualization, phylogenetic inference, analysis and sharing of minimum spanning trees. Nucleic Acids Research 44, W246-W251 (2016).

20. Giannouli, S. et al. Detection of mutations in the FemXAB protein family in oxacillin-susceptible mecA-positive Staphylococcus aureus clinical isolates. Journal of Antimicrobial Chemotherapy 65, 626-633 (2010).

21. Ghosh, C. et al. Aryl-Alkyl-Lysines: Agents That Kill Planktonic Cells, Persister Cells, Biofilms of MRSA and Protect Mice from Skin-Infection. PLOS ONE 10, e0144094 (2015).

22. Stepanović, S., Vuković, D., Dakić, I., Savić, B. \& Švabić-Vlahović, M. A modified microtiter-plate test for quantification of staphylococcal biofilm formation. Journal of Microbiological Methods 40, 175-179 (2000).

23. Bagga, B., Reddy, A. K. \& Garg, P. Decreased susceptibility to quinolones in methicillin-resistant Staphylococcus aureus isolated from ocular infections at a tertiary eye care centre. British Journal of Ophthalmology 94, 1407-1408 (2010).

24. Hsiao, C.-H. et al. Methicillin-Resistant Staphylococcus aureus Ocular Infection: A 10-Year Hospital-Based Study. Ophthalmology 119, 522-527 (2012).

25. Vola, M. E. et al. Prevalence and antibiotic susceptibility of methicillin-resistant Staphylococcus aureus in ocular infections. Arquivos Brasileiros de. Oftalmologia 76, 350-353 (2013).

26. Kang, Y.-C. et al. Methicillin-Resistant Staphylococcus aureus Ocular Infection in Taiwan: Clinical Features, Genotying, and Antibiotic Susceptibility. Medicine 94, e1620 (2015).

27. Elhassan, M. M., Ozbak, H. A., Hemeg, H. A., Elmekki, M. A. \& Ahmed, L. M. Absence of the mec A Gene in Methicillin Resistant Staphylococcus aureus Isolated from Different Clinical Specimens in Shendi City, Sudan. BioMed Research International 2015, 1-5 (2015).

28. Becker, K. et al. Detection of mecA - and mecC -Positive Methicillin-Resistant Staphylococcus aureus (MRSA) Isolates by the New Xpert MRSA Gen 3 PCR Assay: Table 1. Journal of Clinical Microbiology 54, 180-184 (2016).

29. Sharff, K. A. et al. Genotypic Resistance Testing Creates New Treatment Challenges: Two Cases of Oxacillin-Susceptible MethicillinResistant Staphylococcus aureus. Journal of Clinical Microbiology 50, 4151-4153 (2012).

30. Phaku, P. et al. Unveiling the molecular basis of antimicrobial resistance in Staphylococcus aureus from the Democratic Republic of the Congo using whole genome sequencing. Clinical Microbiology and Infection 22, 644.e1-644.e5 (2016).

31. Bergerbachi, B. \& Tschierske, M. Role of fem factors in methicillin resistance. Drug Resistance Updates 1, 325-335 (1998).

32. Li, X. et al. The role of femA regulating gene on methicillin-resistant Staphylococcus aureus clinical isolates. Médecine et Maladies Infectieuses 42, 218-225 (2012).

33. Coppens, J. et al. Novel composite SCCmec type III element in ST239 MRSA isolated from an Indian hospital. Journal of Antimicrobial Chemotherapy, https://doi.org/10.1093/jac/dky399 (2018).

34. Neetu, T. P. \& Murugan, S. Genotyping of methicillin resistant staphylococcus aureus from tertiary care hospitals in Coimbatore, South India. Journal of Global Infectious Diseases 8, 68 (2016).

35. D’Souza, N., Rodrigues, C. \& Mehta, A. Molecular Characterization of Methicillin-Resistant Staphylococcus aureus with Emergence of Epidemic Clones of Sequence Type (ST) 22 and ST 772 in Mumbai, India. Journal of Clinical Microbiology 48, 1806-1811 (2010).

36. Feil, E. J. et al. Rapid Detection of the Pandemic Methicillin-Resistant Staphylococcus aureus Clone ST 239, a Dominant Strain in Asian Hospitals. Journal of Clinical Microbiology 46, 1520-1522 (2008).

37. Bouchiat, C. et al. Epidemiology of Staphylococcus aureus in Bangalore, India: emergence of the ST217 clone and high rate of resistance to erythromycin and ciprofloxacin in the community. New Microbes and New Infections 7, 15-20 (2015).

38. Oyama, T. et al. Biofilm-Forming Methicillin-Resistant Staphylococcus aureus Survive in Kupffer Cells and Exhibit High Virulence in Mice. Toxins 8, 198 (2016).

39. Cha, J.-O., Park, Y.-K., Lee, Y. S. \& Chung, G. T. In vitro biofilm formation and bactericidal activities of methicillin-resistant Staphylococcus aureus clones prevalent in Korea. Diagnostic Microbiology and Infectious Disease 70, 112-118 (2011).

40. William da Fonseca Batistão, D. et al. Biofilm formation of Brazilian meticillin-resistant Staphylococcus aureus strains: prevalence of biofilm determinants and clonal profiles. Journal of Medical Microbiology 65, 286-297 (2016).

41. Dhiman, M., Bhatt, N., Chau-Han, V., Farooq, U. \& Khan, A. Emergence of methicillin resistant Staphylococcus aureus (MRSA) isolates from north India harboring a novel sasX gene: further analyzing its role in biofilm formation. Ann Med. Biomed Sci 3, 46-50 (2017).

42. Brennan, G. I. et al. Emergence of Hospital- and Community-Associated Panton-Valentine Leukocidin-Positive MethicillinResistant Staphylococcus aureus Genotype ST772-MRSA-V in Ireland and Detailed Investigation of an ST772-MRSA-V Cluster in a Neonatal Intensive Care Unit. Journal of Clinical Microbiology 50, 841-847 (2012).

43. McCarthy, H. et al. Methicillin resistance and the biofilm phenotype in Staphylococcus aureus. Frontiers in Cellular and Infection Microbiology 5 (2015). 
44. Cafiso, V. et al. agr-Genotyping and transcriptional analysis of biofilm-producing Staphylococcus aureus. FEMS Immunology \& Medical Microbiology 51, 220-227 (2007).

45. Pozzi, C. et al. Methicillin Resistance Alters the Biofilm Phenotype and Attenuates Virulence in Staphylococcus aureus DeviceAssociated Infections. PLoS Pathogens 8, e1002626 (2012).

46. Getahun, E., Gelaw, B., Assefa, A., Assefa, Y. \& Amsalu, A. Bacterial pathogens associated with external ocular infections alongside eminent proportion of multidrug resistant isolates at the University of Gondar Hospital, northwest Ethiopia. BMC Ophthalmology 17 (2017).

47. Suriyanarayanan, T. et al. Quantitative Proteomics of Strong and Weak Biofilm Formers of Enterococcus faecalis Reveals Novel Regulators of Biofilm Formation. Molecular \& Cellular Proteomics 17, 643-654 (2018).

\section{Acknowledgements}

V.B. acknowledge the Department of Science and Technology- India (2016/DST/INSPIRE/04/2015/000242) and Science and Engineering Research Board extramural grant (EMR/2016/007266) for its financial support.

\section{Author contributions}

V.B. designed the study. U.B., P.S., S.M., S.C., S.N.A. and V.B. have done all the experiments. Data compiling and manuscript writing was done by V.B. P.S. and S.S. have helped in data compiling, analysis and manuscript editing.

\section{Competing interests}

The authors declare no competing interests.

\section{Additional information}

Supplementary information is available for this paper at https://doi.org/10.1038/s41598-019-52557-z.

Correspondence and requests for materials should be addressed to V.B.

Reprints and permissions information is available at www.nature.com/reprints.

Publisher's note Springer Nature remains neutral with regard to jurisdictional claims in published maps and institutional affiliations.

Open Access This article is licensed under a Creative Commons Attribution 4.0 International ense, which permits use, sharing, adaptation, distribution and reproduction in any medium or format, as long as you give appropriate credit to the original author(s) and the source, provide a link to the Creative Commons license, and indicate if changes were made. The images or other third party material in this article are included in the article's Creative Commons license, unless indicated otherwise in a credit line to the material. If material is not included in the article's Creative Commons license and your intended use is not permitted by statutory regulation or exceeds the permitted use, you will need to obtain permission directly from the copyright holder. To view a copy of this license, visit http://creativecommons.org/licenses/by/4.0/.

(c) The Author(s) 2019 